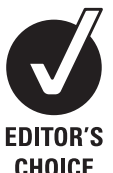

CHOICE

\title{
Risk of autism spectrum disorders in children born after assisted conception: a population-based follow-up study
}

\author{
D Hvidtjørn, ${ }^{1}$ J Grove, ${ }^{1}$ D Schendel, ${ }^{2}$ L A Schieve, ${ }^{2}$ C Sværke, ${ }^{1}$ E Ernst, ${ }^{3}$ P Thorsen ${ }^{1}$
}

1 Institute of Public Health at the Department of Epidemiology, University of Aarhus, Aarhus, Denmark

${ }^{2}$ Centers for Disease Control and Prevention, National Center on Birth Defects and

Developmental Disabilities, Atlanta, Georgia, USA

${ }^{3}$ Fertility Section, Department of Obstetrics and Gynaecology, Aarhus University Hospital, Aarhus, Denmark

\section{Correspondence to}

Dr Dorte Hvidtjørn, Institute of

Public Health, at the Department of Epidemiology, University of Aarhus, Paludan-Müllers Vej 17, 8000 Aarhus C, Denmark: dh@soci.au.dk

The findings and conclusions in this report are those of the authors and do not necessarily represent the official position of the Centers for Disease Control and Prevention.

Accepted 30 December 2009 Published Online First 27 June 2010

\section{ABSTRACT}

Objectives To assess the risk of autism spectrum disorders (ASD) in children born after assisted conception compared with children born after natural conception.

Design Population-based follow-up study.

Setting All children born alive in Denmark 1995-2003. Participants 588967 children born in Denmark from January 1995 to December 2003. Assisted conception was defined as in vitro fertilisation (IVF) with or without intracytoplasmic sperm injection and ovulation induction (OI) with or without subsequent insemination. Children exposed to IVF or OI were identified in the IVF Register and in the Danish Drug Prescription Register.

Main outcome measures $A$ diagnosis of $A S D$ in the Danish Psychiatric Central Register

Results 33139 (5.6\%) of all children born in Denmark in 1995-2003 resulted from assisted conception, 225 of whom (0.68\%) had a diagnosis of ASD. Of the 555828 children born in this period after natural conception, 3394 $(0.61 \%)$ had a diagnosis of ASD. The follow-up time was 4-13 years (median 9 years). In crude analyses, children born after assisted conception had an increased risk of a diagnosis of ASD: crude hazard rate ratio (HRR) 1.25 (95\% Cl 1.09 to 1.43). In analyses adjusting for maternal age, educational level, parity, smoking, birth weight and multiplicity, the risk disappeared: adjusted HRR 1.13 (95\% Cl 0.97 to 1.31). However, subgroup analyses that suggest possible associations in women who received follicle stimulating hormone indicate the need for further study.

Discussion This population-based follow-up study found no risk of $A S D$ in children born after assisted conception.

\section{INTRODUCTION}

The aetiology behind infantile autism and autism spectrum disorders (ASD) is multifactorial and largely unknown. ${ }^{1}$ Associations between ASD and assisted conception might be anticipated because of at least three shared risk factors: high parental age $^{2-7}$ high maternal educational level ${ }^{3} 8$ and hormonal disturbances. ${ }^{9-11}$ Furthermore, a higher risk of ASD in children born after assisted conception might be expected since assisted conception leads to a high rate of preterm delivery (PTD) and low birth weight (BW), ${ }^{12-18}$ and associations between PTD or low BW and ASD have been reported. ${ }^{3} 51920$ The use of assisted conception has increased over the last two decades. ${ }^{13} 21$

We identified eight studies assessing the risk of infantile autism or ASD in children born after assisted conception. ${ }^{22-29}$ They present inconsistent results and disclose methodological problems such as lack of statistical power ${ }^{25-27}$ or only evaluating a combined group of psychiatric disorders, rather than ASD specifically. ${ }^{27}$ Furthermore, six studies only assessed one type of assisted conception, in vitro fertilisation (IVF). In all, only one study found a significantly higher occurrence of ASD in children born after assisted conception compared with children born after natural conception ${ }^{25}$; however, four other studies reported a non-statistically significantly increased risk. ${ }^{22} 23 \quad 27 \quad 28$ One case-control study reported a protective effect of assisted conception on infantile autism after adjusting for several factors including gestational age. $^{29}$ None of the studies distinguished between different underlying causes of infertility or between different types of medications used in the treatment. Consequently, the relationship between ASD and assisted conception is still not well understood. To overcome methodological problems in former studies, we assessed the risk of infantile autism and ASD in a large population-based cohort of children born after assisted conception. Assisted conception was defined as IVF with or without intracytoplasmic sperm injection (ICSI) and ovulation induction (OI) with or without subsequent in semination. We also evaluated underlying causes of infertility and specific medications used in the treatments.

\section{METHODS}

\section{Study population}

This cohort study was based on data from Danish National Registers and linkage between the registers was achieved by use of the unique registration number given to all citizens in Denmark. Our study comprised all children born alive in Denmark from 1 January 1995 to 31 December 2003, identified through the Danish Medical Birth Register (MBR) which contains information on all births in Denmark. $^{31}$

\section{Exposure}

Children exposed to IVF were identified through the IVF Register which holds data from all private and public fertility clinics including underlying causes of infertility (tubal, male, ovulation or mixed and unspecified factors). ${ }^{32}$ Children exposed to OI were identified through the Danish Drug Prescription Register (DDPR) which holds information on all prescription drugs sold at pharmacies in Denmark. The medications used during OI are prescription drugs bought at the pharmacy, enabling identification of the women who went through OI in the DDPR. Drugs used in assisted conception 
were identified by the authors by cross-checking with the official Danish Pharmaceutical Classified Catalogue, www.medicin.dk, the US website www.drugs.com, books of instruction of Danish fertility clinics from the time period in question and by means of clinical experience. To identify women who had hormonal treatment in relation to the index pregnancy, we set up a time window for the date of dispatch of 12 weeks before and 4 weeks after the last menstrual period (LMP). As the drugs used in OI are also used in IVF, we excluded women from the OI group who were included in the IVF Register with the same LMP date.

\section{Outcome}

Children with a diagnosis of ASD (F84.0, F84.1, F84.5, F84.8 and F84.9) or, specifically, infantile autism (F84.0) up to 8 May 2008 were identified via the Danish Psychiatric Central Register (DPCR). The DPCR contains information on all Danish psychiatric inpatient and outpatient admissions since $1995,{ }^{33}$ and in Denmark all autism diagnoses are made at public child mental health services, reporting all inpatient and outpatient discharge diagnoses to the DPCR.

\section{Covariates}

Further covariate information was obtained from MBR and Statistics Denmark: BW, gestational age, multiplicity, sex, maternal age, educational level, smoking and parity. Maternal age was divided into 5-year age groups: 20-24, 25-29 (referent category), 30-34, 35-39, 40+; BW was grouped into 500-1499 g, 1500-2499 g and normal BW $\geq 2500 \mathrm{~g}$ (referent category); education level was categorised as low (9-10 years of education), intermediate (11-16 years of education) (referent category) and high ( $\geq 17$ years of education); parity was dichotomised into primiparous and multiparous (referent category); multiplicity was divided into singletons (referent category) and multiples; and maternal smoking during pregnancy was grouped into non-smoker (referent category) and smoking plus 'stopped smoking during pregnancy' combined. As there were no mothers aged $<20$ years in the IVF Register, 10137 children (1.7\%) born to mothers aged $<20$ years were excluded.

\section{Multivariate analyses}

Cox regression was used to measure the risk of an ASD diagnosis in children born after assisted conception combined and in IVF and OI separately. We conducted multivariate analyses including maternal age, parity, smoking, educational level, BW and multiplicity. Owing to the skewed sex distribution in ASD, analyses were conducted for all children and stratified by sex.

\section{Sub-analyses}

In further analyses we explored if the risk of ASD was associated with a single underlying cause of infertility (tubal, male, ovulation or mixed and unspecified factors), but this information was only available in the IVF Register and only for 9855 (66\%) of the IVF children as many of the IVF children had unspecific or more than one cause. Finally, to examine if different hormones used in assisted conception (downregulating hormones, clomiphene, follicle stimulating hormone (FSH) and human chorionic gonadotropin (hCG)) were associated with ASD, we made exploratory analyses with specific hormone exposure. Information about the use of specific hormones was available from the DDPR for all OI children but only for $51 \%$ of the IVF children. This was due to different dispensing procedures at IVF clinics; some clinics dispense the medication directly to the women while other IVF clinics provide prescriptions for the pharmacy. Only the latter group of women is included in the DDPR.

\section{Statistical analyses}

The risk of a diagnosis of ASD or infantile autism was measured by the incidence rate, using the child as the unit of analysis. The incidence rate was defined as the number of children diagnosed with ASD in a given time frame (1 January 1995 to 8 May 2008) divided by the total number of person-years the children in the cohort were at risk for the diagnosis during that time. We used Cox regression and the incidence rate ratio was estimated by the hazard rate ratio (HRR) with 95\% CIs. It was verified that the proportional hazards assumption was met. To account for correlations between siblings in the cohort, additional analyses were done using robust standard errors. To reduce bias that might arise if exposed children at high risk for ASD were more likely to die as infants than unexposed children, we conducted additional analyses excluding children aged $<1$ year (ie, limiting the analysis to infant survivors).

Stata Version 8.2 (StataCorp, College Station, Texas, ISA) was used for the analyses.

\section{RESULTS}

From 1 January 1995 to 31 December 2003, 588967 children were born alive in Denmark to 399598 mothers aged $\geq 20$ years. In this period, $33139(5.6 \%)$ children resulted from assisted conception, 14991 (2.6\%) from IVF and 18148 (3.0\%) from OI. Compared with mothers who conceived naturally, those with assisted conception were statistically significantly older $(p<0.001)$, more often primiparous $(p<0.001)$, non-smokers $(p<0.001)$, had higher educational level $(p=0.002)$ and delivered more multiples $(p<0.001)$, children with low BW $(p<0.001)$ and PTD $(p<0.001)$ (table 1). Furthermore, the difference in most of these characteristics from mothers who conceived naturally was greater for mothers having IVF than for those having OI (table 1).

Up to 8 May 2008, 3619 (0.61\%) children from the cohort were diagnosed with ASD; a diagnosis of infantile autism was given to 1385 children (0.24\%). For all diagnoses, the follow-up time was $4-13$ years (median 9 years). Within the time period 1995-2003, 3479 mothers had one child with a diagnosis of ASD, 64 mothers had two children with an ASD diagnosis and 4 mothers had three children with a diagnosis of ASD. Mothers of children with ASD were significantly different from mothers of other children in selected characteristics: they were older $(p=0.002)$, more often primiparous $(p<0.001)$, smokers $(p<0.001)$, they had a higher or lower educational level $(p<0.001)$, more preterm deliveries $(\mathrm{p}<0.001)$ and children with low BW $(\mathrm{p}<0.001)$ (table 2).

In children born after assisted conception, the risk of having a diagnosis of ASD or infantile autism was increased (crude HRR 1.25 (95\% CI 1.09 to 1.43 ) and 1.25 (95\% CI 1.01 to 1.54$)$, respectively). The crude HRR for the risk of a diagnosis of ASD was 1.18 (95\% CI 0.95 to 1.44) for children born after IVF and 1.31 (95\% CI 1.10 to 1.56) for those born after OI. In analyses adjusted for maternal age, educational level, smoking, parity, BW and multiplicity, we found a HRR of 1.13 (95\% CI 0.97 to 1.31 ) in the combined assisted conception group and higher risk estimates in OI than in IVF (table 3). In the adjusted analyses, only girls born after OI had a statistically significant increased risk of ASD.

Similar results were seen for infantile autism but with wider CIs (data not shown). The risk of ASD for boys and girls did not differ significantly. Restricting the analyses to infants who survived their first year showed similar results to the full cohort (data not shown). Dropping variables from the adjusted analyses 
Table 1 Characteristics of women who used assisted conception and their children, Denmark 1995-2003

\begin{tabular}{|c|c|c|c|c|c|c|}
\hline & Natural conception & Assisted conception & p Value & IVF & 이 & p Value \\
\hline Maternal age & & & $<0.001$ & & & $<0.001$ \\
\hline $20-24$ & $81371(14.6)$ & $1175(3.6)$ & & $206(1.4)$ & $969(5.39)$ & \\
\hline $25-29$ & $211078(38.0)$ & $8423(25.4)$ & & 2822 (18.8) & $5601(30.9)$ & \\
\hline $35-39$ & $66776(12.0)$ & $8174(24.7)$ & & $4547(30.3)$ & $3627(20.0)$ & \\
\hline $40+$ & $9651(1.8)$ & $1182(3.5)$ & & $625(4.2)$ & $557(3.1)$ & \\
\hline Multiparous & $327746(58.9)$ & $10968(33.1)$ & & $3892(26.0)$ & $7076(39.0)$ & \\
\hline Educational level (years) & & & $<0.001$ & & & 0.002 \\
\hline Lower $(9-10)$ & $111404(23.1)$ & $4641(16.7)$ & & $2175(17.5)$ & $2466(16.1)$ & \\
\hline Intermediate (11-16) & $331842(68.7)$ & $20187(72.7)$ & & $8975(72.3)$ & $11212(73.1)$ & \\
\hline Higher $(17+)$ & $39663(8.2)$ & $2930(10.6)$ & & $1261(10.2)$ & $1669(10.8)$ & \\
\hline Smoker or stopped in pregnancy & $123512(22.3)$ & $5386(16.3)$ & & $2631(17.6)$ & $2755(15.2)$ & \\
\hline Multiplicity & & & $<0.001$ & & & $<0.001$ \\
\hline Singletons & 541616 (97.6) & $23483(70.9)$ & & $8501(56.7)$ & $14982(82.6)$ & \\
\hline Twins & $13376(2.4)$ & $9120(27.5)$ & & $6234(41.6)$ & $2886(15.9)$ & \\
\hline Triplets & $215(0.0)$ & $495(1.5)$ & & $239(1.6)$ & $256(1.4)$ & \\
\hline Quadruplets & $4(0.0)$ & $17(0.1)$ & & $5(0.1)$ & $12(0.1)$ & \\
\hline Gestational age (weeks) & & & $<0.001$ & & & $<0.001$ \\
\hline $20-27$ & $1247(0.2)$ & $397(1.2)$ & & $278(1.9)$ & $119(0.7)$ & \\
\hline $28-31$ & $3275(0.6)$ & $868(2.6)$ & & $529(3.6)$ & $339(1.9)$ & \\
\hline $32-36$ & $26319(4.8)$ & $5177(15.7)$ & & $3108(20.8)$ & $2069(11.4)$ & \\
\hline
\end{tabular}

Data shown as numbers (percentages). Natural conception compared with the combined assisted conception group and in vitro fertilisation (IVF) compared with ovulation induction (0I).

one at a time showed that the increased risk in the crude analyses seemed to be explained by maternal age and parity but not by smoking, multiplicity, BW and educational level. The HRR in an adjusted analyses not including maternal age was 1.24 (95\% CI 1.06 to 1.44 ) and in an adjusted model without parity the HRR was 1.28 (95\% CI 1.10 to 1.49$)$.

Information on a single underlying cause of infertility was available for 9855 children born after IVF. The risk of ASD was highest for those children with a maternal infertility diagnosis of ovulation factor; however, estimates are imprecise owing to the small sample sizes (table 4).

Information on specific hormonal medications used in assisted conception was available for 25867 children and, in the analyses of the risk of ASD by different hormone exposure, the association with ASD was strongest with medications containing FSH and not clearly evident for other hormonal medications (table 5). Divided into specific treatment type, the FSH associations were observed only within IVF treatments. The risk was higher for girls than for boys for all hormones and statistically significant for FSH, hCG and clomiphene. We were able to assess FSH use in all OI treatments but only in $51 \%$ of the IVF treatments as we could not confirm the specific medications used through the DDPR for the remaining IVF treatments. Women who under- went IVF and had detailed medication data from DDPR were different from those without DDPR data in that they were older, more often multiparous, non-smokers, more highly educated and more likely to have been treated at a private rather than a public clinic. They were also more likely to have ovulation factor infertility; however, even with this increase, it is important to note that, while the majority of women who underwent IVF and had detailed medication data from DDPR used FSH $(85 \%)$, in only a small minority (9\%) was ovulation factor infertility documented. We were unable to assess both factors simultaneously because of limited sample size (only 58 cases had data on both the single underlying cause of infertility and specific hormone treatment).

\section{DISCUSSION}

\section{Main results}

In this large population-based cohort study we found no risk of ASD in children born after assisted conception (adjusted HRR 1.13 (95\% CI 0.97 to 1.31)). The increased risk for ASD observed in unadjusted analyses seemed to be related to high maternal age and parity but did not appear to be related to educational level, low BW, smoking or multiplicity. This is the 
Table 2 Characteristics of children with an ASD diagnosis and their mothers, Denmark 1995-2003

\begin{tabular}{lcrr}
\hline & No ASD & ASD & p Value \\
\hline Maternal age & & & $<0.001$ \\
$20-24$ & $81996(14.0)$ & $550(15.2)$ & \\
$25-29$ & $218242(37.3)$ & $1259(34.8)$ & \\
$30-34$ & $199901(34.2)$ & $1218(33.7)$ & \\
$35-39$ & $74442(12.7)$ & $508(14.0)$ & \\
$40+$ & $10749(1.8)$ & $84(2.3)$ &
\end{tabular}

Parity

Primiparous

Multiparous

$248429(42.4) \quad 1801(49.8)$

$336896(57.6) \quad 1818(50.2)$

Educational level (years)

Lower (9-10)

Intermediate (11-16)

$115139(22.7)$

$349884(69.0)$

$906(27.0)$

Higher $(17+)$

$42283(8.3)$

$2145(63.8)$

310 (9.2)

\section{Smoking}

Non-smoker

$454685(78.0) \quad 2713(75.3)$

Smoker or stopped in pregnancy

$128009(22.0)$

$889(24.7)$

Multiplicity

Singletons

Twins

Triplets

Quadruplets

561598 (96.1)

22389 (3.8)

$703(0.1)$

$21(0.0)$

3501 (96.9)

107 (3.0)

$7(0.1)$

$0(0.0)$

Gestational age (weeks)

$\begin{array}{lcc}20-27 & 1624(0.3) & 20(0.5) \\ 28-31 & 4114(0.7) & 29(0.8) \\ 32-36 & 31268(5.4) & 228(6.4) \\ 37-41 & 496232(85.3) & 2996(83.5) \\ 42+ & 48158(8.3) & 315(8.8)\end{array}$

Birth weight $(\mathrm{g})$

$\begin{array}{lrr}500-1499 & 500(0.9) & 44(1.2) \\ 1500-2499 & 24414(4.2) & 196(5.5) \\ 2500+ & 550889(94.9) & 3346(93.3)\end{array}$

$<0.001$

Data shown as numbers (percentages). Children without autism spectrum disorders (ASD) compared with children with ASD.

largest population-based study to date, with 33139 children exposed to assisted conception, and hence the first study also permitting assessment of different types of assisted conception separately. In the present study the prevalence of ASD was 6 per 1000 children, similar to reports from other studies where a prevalence of ASD of 4.7, 5.8 and 6-7 per 1000 has been reported. ${ }^{34-36}$

\section{Sex, maternal age, parity and birth weight}

The risk from assisted conception tended to be higher in girls than in boys in all groups. Other studies suggest that the aetiological profile behind ASD in girls and boys may be different. ${ }^{37}$ Furthermore, since the a priori risk in boys is so much higher than the risk in girls, it is possible that ASD in boys represents either a more heterogeneous mix of autism aetiological subtypes or a mix of potential aetiologies which could moderate an association of assisted conception with ASD.

The risk of autism or ASD is associated with advanced maternal age in some studies with adjusted analyses, ${ }^{2-4}$ but not in all. ${ }^{5} 1920$ In our adjusted analysis the risk of ASD from assisted conception was no longer statistically significant when controlling for maternal age (table 3). Thus, the increased risk of ASD in assisted conception seems to be partly explained by high maternal age.

There is also a higher risk of ASD in first-born children in some studies, ${ }^{2-4}$ but not in others. ${ }^{51920}$ A recent meta-analysis found an increased risk in both first-born and subsequent children (third and over). ${ }^{38}$ In our analyses the risk of ASD from assisted conception did not remain statistically significant when we adjusted for parity, suggesting that the increased risk of ASD in the crude analyses is also partly explained by a large proportion of primiparous mothers having assisted conception.

In assisted conception more children are born with low BW and, in general, the risk of ASD increases with decreasing BW in most studies, ${ }^{3} 51920$ although not in one smaller study by Glasson et al using mean BW. ${ }^{4}$ In our study, low BW did not seem to explain the increased risk of ASD in assisted conception. This is in contrast to studies of cerebral palsy where the increased risk of cerebral palsy in children born after IVF is partly explained by multiplicity and PTD, factors leading to a high proportion of low BW children. ${ }^{30} 39$

\section{Other studies}

Our results contradict previous studies which found a higher occurrence of ASD in children born after assisted conception. 2223252728 One case-control study showed a protective association between a general notation of assisted conception on the birth record and infantile autism. ${ }^{29}$ The possibility of biased exposure ascertainment cannot be ruled out in the latter case-control study.

\section{Type of assisted conception}

There was a tendency for higher risk estimates in the OI group than in the IVF group, but the differences in risk between the two types of treatment were not statistically significant. However, this trend was consistent in both boys and girls with ASD and might be explained by competing risk factors in the IVF group, as this group is at higher risk of ASD a priori owing to higher maternal age, more multiple pregnancies and more primiparous mothers (table 1). However, the risk of ASD was not statistically significant in the adjusted analyses in either type of assisted conception.

Table 3 Crude and adjusted hazard rate ratios and $95 \%$ Cls for the association between ASD and assisted conception*

\begin{tabular}{|c|c|c|c|c|c|c|}
\hline & \multicolumn{2}{|l|}{ All } & \multicolumn{2}{|l|}{ Boys } & \multicolumn{2}{|l|}{ Girls } \\
\hline & Crude & Adjusted † & Crude & Adjusted † & Crude & Adjusted $\dagger$ \\
\hline Assisted conception & 1.25 (1.09 to 1.43$)$ & $1.13(0.97$ to 1.31$)$ & 1.18 (1.01 to 1.37$)$ & 1.07 (0.91 to 1.27$)$ & 1.55 (1.14 to 2.10$)$ & $1.32(0.93$ to 1.88$)$ \\
\hline 이 & 1.31 (1.10 to 1.56$)$ & $1.20(0.99$ to 1.44$)$ & $1.20(0.99$ to 1.47$)$ & $1.12(0.91$ to 1.38$)$ & 1.80 (1.24 to 2.61$)$ & 1.56 (1.03 to 2.36$)$ \\
\hline
\end{tabular}

*Reference: natural conception.

†Adjusted for maternal age, educational level, parity, smoking, body weight and multiplicity.

ASD, autism spectrum disorders; IVF, in vitro fertilisation; OI, ovulation induction. 
Table 4 Crude hazard rate ratios (HRRs) and 95\% Cls for the association between ASD and IVF according to specific underlying cause of infertility

\begin{tabular}{lrrl}
\hline Underlying factor & $\begin{array}{l}\text { Numbers } \\
\text { exposed }\end{array}$ & $\begin{array}{l}\text { ASD } \\
\text { Numbers } \\
\text { exposed }\end{array}$ & Crude HRR \\
\hline Natural conception & 555828 & $3.394(0.61 \%)$ & Reference \\
Mixed or unspecific factors & 5136 & $39(0.76 \%)$ & $1.49(1.08$ to 2.04) \\
Tubal factor & 4859 & $26(0.54 \%)$ & $0.86(0.59$ to 1.27$)$ \\
Ovulation factor & 929 & $7(0.75 \%)$ & $1.63(0.78$ to 3.42$)$ \\
Male factor & 4019 & $23(0.57 \%)$ & $1.18(0.79$ to 1.78$)$ \\
\hline
\end{tabular}

Percentages within categories are given

ASD, autism spectrum disorders; IVF, in vitro fertilisation.

\section{Hormonal exposure}

In adjusted analyses an increased risk of ASD from FSH exposure was seen (HRR 1.44 (95\% CI 1.16 to 1.80)). The risk was statistically significant in both boys and girls but, when specific treatments were considered, the risk was only statistically significant in IVF. Hormonal disorders could link assisted conception with ASD as some underlying causes of infertility are hormone-related and hormonal medications are used as part of infertility treatments. Furthermore, various theories for associations between abnormal levels of sex hormones and ASD have been presented, based on reports of increased levels of fetal testosterone in children with ASD, ${ }^{9}$ women with ASD having significantly more polycystic ovary syndrome, delayed puberty and irregular menstrual cycles in adulthood than controls, ${ }^{10}$ and children with ASD having significantly lower levels of serum FSH and significantly higher levels of serum total testosterone relative to normal reference ranges. ${ }^{11}$ Our findings concerning the risk of ASD following specific hormone exposure in assisted conception should be interpreted with caution. In treatment regimes in assisted conception, hormones are used in various combinations and hormone exposure groups therefore overlap. We did not have data to assess specific medication dosages or protocols. However, we were able to assess FSH use in all OI treatments but only in $51 \%$ of the IVF treatments as we could not confirm the specific medications used through the DDPR for the remaining IVF treatments. We therefore could not assess medication use in the context of the underlying fertility condition being treated. It is therefore unclear whether it is factors linked to use of FSH (underlying cause of infertility or other conditions), a teratogenic effect from FSH medication per se, the combined effect of exogenous FSH on the endogenous hormonal profile or some perhaps yet unmeasured factor which underlies the association between FSH medications and ASD. The risk of ASD in different hormone regimes would best be assessed in a randomised controlled trial, but this possibility is ruled out by the infrequency of ASD. Observational studies such as this are therefore the only alternative, but our findings indicate the need for further more specific investigations of possible associations between exposure to exogenous FSH and ASD.

\section{Strengths and limitations of the study}

Our sources of data were Danish National Health Registers. These registers are maintained for administrative or monitoring purposes and will therefore not always include specific details desirable for research. For exposure to IVF we used the IVF Register and, according to the Danish legislation, it is mandatory for all public and private fertility clinics in Denmark to report all initiated IVF and ICSI treatments, successful or not, to the IVF Register and the register is therefore considered almost complete. ${ }^{32}$ For exposure to OI we used the DDPR with data on all dispatched prescriptions for hormones used in OI. The main purpose of the DDPR is entirely administrative, that is to ensure that the prescription is not used twice, and therefore it is expected that all dispatches are reported to the DDPR. However, it was not possible to establish the date on which the woman actually administered the medicine. Hormones used in OI can be prescribed for the next three cycles and, consequently, we had to select a time window of prescriptions within 3 months before LMP to define the use of OI in the index pregnancy. Therefore, the hormones could have been administered 1 or 2 months earlier than the index pregnancy. In addition, we cannot be sure that the woman ever administered the medicine she purchased and therefore the findings regarding $\mathrm{OI}$ have to be interpreted with caution. However, we have no reason to believe such exposure misclassification would be related to ASD so, to the extent that we erroneously included some children born by natural conception in the OI category, our results might actually be attenuated The quality of autism diagnoses found in the DPCR has been validated by experts ${ }^{40}$; after evaluating almost 500 medical records of children registered with infantile autism in the DPCR, $94 \%$ of the children met the criteria for a correct diagnosis.

As a number of children were followed for $<6$ years, some children with ASD may not have been diagnosed before the end of follow-up. Also, since the number and proportion of assisted conceptions increased over the study period, a disproportionate number of children born after assisted conception had a shorter follow-up time than naturally conceived children. To deal with these aspects of the data we used Cox regression models in the statistical analysis which account for differences in follow-up time.

\section{CONCLUSION}

In conclusion, we found no overall increased risk of ASD in children born after assisted conception. However, in sub-analyses we did find an increased risk in a subgroup of women who had OI treatment and were exposed to FSH. Additional studies with more detailed information on underlying causes of infertility and use of specific hormonal medications may help to pinpoint the underlying mechanism of this increased risk.

Table 5 Hazard rate ratios and $95 \%$ Cls for the association between ASD and assisted conception combined and divided into IVF and OI according to specific hormones*

\begin{tabular}{lllll}
\hline & Assisted conception, all & Assisted conception, boys & Assisted conception, girls & IVF, all \\
\hline Downregulation & $0.82(0.49$ to 1.37$)$ & $0.79(0.45$ to 1.40$)$ & $0.93(0.29$ to 2.92$)$ & $1.99(1.23$ to 3.18$)$ \\
FSH & $1.44(1.16$ to 1.80$)$ & $1.32(1.02$ to 1.70$)$ & $1.85(1.23$ to 2.77$)$ & $1.09(0.48$ to 2.51$)$ \\
hCG & $1.28(1.05$ to 1.55$)$ & $1.16(0.93$ to 1.44$)$ & $1.75(1.15$ to 2.65$)$ & $1.29(0.89$ to 1.89$)$ \\
Clomiphene & $1.14(0.93$ to 1.40$)$ & $1.02(0.80$ to 1.29$)$ & 1.10 to 2.71$)$
\end{tabular}

*Reference: natural conception, adjusted for maternal age, education, parity, smoking, body weight and multiplicity.

ASD, autism spectrum disorders; FSH, follicle stimulating hormone; hCG, human chorionic gonadotropin; IVF, in vitro fertilisation; Ol, ovulation induction. 


\section{What is already known on this subject}

The few studies that have assessed the association between autism spectrum disorders (ASD) and assisted conception present inconsistent results and disclose methodological limitations such as lack of statistical power.

- Associations between ASD and assisted conception might be anticipated owing to at least three shared risk factors: high parental age, high maternal educational level and hormonal disturbances.

- A higher risk of ASD in children born after assisted conception might also be expected since assisted conception leads to a high rate of preterm delivery and low birth weight, and associations between preterm delivery or low birth weight and ASD have been reported.

\section{What this study adds}

- This population-based follow-up study found no risk of ASD in children born after assisted conception.

- However, we did find an increased risk in a subgroup of women who had IVF treatment and were exposed to follicle stimulating hormone.

Funding The study was funded as a co-financed PhD project by The Danish Agency for Science, Technology and Innovation, University of Aarhus and The Elsass Foundation. Further funding was supplied by Sofiefonden, The Health Insurance Foundation, The Augustinus Foundation, Julie von Müllens Foundation, Direktør Jacob Madsen and Hustru Olga Madsens Fond and Aase and Ejnar Danielsen Foundation.

Competing interests None.

Ethics approval Approval for this study was obtained from the Danish Data Protection Agency.

Contributors DH, PT and JG were responsible for conception and design. DH, JG and CS conducted the statistical analyses and the interpretation of data. DH wrote the first draft of the manuscript. All authors provided critical input at all stages and critically reviewed and contributed to the final draft. PT and DH are guarantors of the study. All authors had full access to the data.

Provenance and peer review Not commissioned; externally peer reviewed.

\section{REFERENCES}

1. Baron IS. Autism spectrum disorder: complex, controversial, and confounding. Neuropsychol Rev 2008;18:271-2

2. Durkin MS, Maenner MJ, Newschaffer CJ, et al. Advanced parental age and the risk of autism spectrum disorder. Am J Epidemiol 2008;168:1268-76.

3. Croen LA, Grether JK, Selvin S. Descriptive epidemiology of autism in a California population: who is at risk? J Autism Dev Disord 2002;32:217-24.

4. Glasson EJ, Bower C, Petterson B, et al. Perinatal factors and the development of autism: a population study. Arch Gen Psychiatry 2004;61:618-27.

5. Larsson HJ, Eaton WW, Madsen KM, et al. Risk factors for autism: perinatal factors, parental psychiatric history, and socioeconomic status. Am J Epidemiol 2005;161:916-25.

6. Lauritsen MB, Pedersen CB, Mortensen PB. Effects of familial risk factors and place of birth on the risk of autism: a nationwide register-based study. J Child Psychol Psychiatry 2005; 46:963-71.

7. Reichenberg A, Gross R, Weiser M, et al. Advancing paternal age and autism. Arch Gen Psychiatry 2006;63:1026-32.

8. Bhasin TK, Schendel D. Sociodemographic risk factors for autism in a US metropolitan area. J Autism Dev Disord 2007;37:667-77.
9. Auyeung B, Baron-Cohen S, Ashwin E, et al. Fetal testosterone and autistic traits. Br J Psychol 2009;100:1-22.

10. Ingudomnukul E, Baron-Cohen $\mathrm{S}$, Wheelwright $\mathrm{S}$, et al. Elevated rates of testosterone-related disorders in women with autism spectrum conditions. Horm Behav 2007; 51:597-604.

11. Geier DA, Geier MR. A clinical and laboratory evaluation of methionine cycletranssulfuration and androgen pathway markers in children with autistic disorders. Horm Res 2006;66:182-8

12. Helmerhorst FM, Perquin DA, Donker D, et al. Perinatal outcome of singletons and twins after assisted conception: a systematic review of controlled studies. BMJ 2004; 328:261.

13. Nyboe AA, Goossens V, Gianaroli L, et al. Assisted reproductive technology in Europe, 2003. Results generated from European registers by ESHRE. Hum Reprod 2007:22:1513-25.

14. Kallen B, Olausson PO, Nygren KG. Neonatal outcome in pregnancies from ovarian stimulation. Obstet Gynecol 2002;100:414-19.

15. Wang JX, Norman RJ, Kristiansson P. The effect of various infertility treatments on the risk of preterm birth. Hum Reprod 2002;17:945-9.

16. Olivennes $\mathbf{F}$, Rufat $P$, Andre $B$, et al. The increased risk of complication observed in singleton pregnancies resulting from in-vitro fertilization (IVF) does not seem to be related to the IVF method itself. Hum Reprod 1993;8:1297-300.

17. Gaudoin M, Dobbie R, Finlayson A, et al. Ovulation induction/intrauterine insemination in infertile couples is associated with low-birth-weight infants. Am J Obstet Gynecol 2003;188:611-16.

18. Ombelet W, Martens G, De Sutter P, et al. Perinatal outcome of 12,021 singleton and 3108 twin births after non-IVF-assisted reproduction: a cohort study. Hum Reprod 2006;21:1025-32

19. Eaton WW, Mortensen PB, Thomsen PH, et al. Obstetric complications and risk for severe psychopathology in childhood. J Autism Dev Disord 2001;31:279-85

20. Hultman CM, Sparen P, Cnattingius S. Perinatal risk factors for infantile autism. Epidemiology 2002:13:417-23.

21. Wright VC, Chang J, Jeng G, et al. Assisted reproductive technology surveillance-United States, 2003. MMWR Surveill Summ 2006:55:1-22

22. Lidegaard 0, Pinborg A, Andersen AN. Imprinting diseases and IVF: Danish Nationa IVF cohort study. Hum Reprod 2005;20:950-4.

23. Ericson A, Nygren KG, Olausson PO, et al. Hospital care utilization of infants born after IVF. Hum Reprod 2002;17:929-32.

24. Stromberg B, Dahlquist G, Ericson A, et al. Neurological sequelae in children born after in-vitro fertilisation: a population-based study. Lancet 2002:359:461-5

25. Pinborg A, Loft A, Schmidt L, et al. Morbidity in a Danish national cohort of 472 IVF/ ICSI twins, 1132 non-IVF/ICSI twins and 634 IVF/ICSI singletons: health-related and social implications for the children and their families. Hum Reprod 2003;18:1234-43.

26. Pinborg A, Loft A, Schmidt L, et al. Neurological sequelae in twins born after assisted conception: controlled national cohort study. BMJ 2004:329:311.

27. Klemetti R, Sevon T, Gissler M, et al. Health of children born as a result of in vitro fertilization. Pediatrics 2006:118:1819-27.

28. Stein D, Weizman A, Ring A, et al. Obstetric complications in individuals diagnosed with autism and in healthy controls. Compr Psychiatry 2006;47:69-75.

29. Maimburg RD, Vaeth M. Do children born after assisted conception have less risk of developing infantile autism? Hum Reprod 2007;22:1841-3.

30. Hvidtjorn D, Schieve L, Schendel D, et al. Cerebral palsy, autism spectrum disorders, and developmental delay in children born after assisted conception: a systematic review and meta-analysis. Arch Pediatr Adolesc Med 2009;163:72-83.

31. Knudsen LB, Olsen J. The Danish Medical Birth Registry. Dan Med Bull 1998:45:320-3.

32. Andersen AN, Westergaard HB, Olsen J. The Danish in vitro fertilisation (IVF) register. Dan Med Bull 1999;46:357-60.

33. Munk-Jorgensen $\mathbf{P}$, Mortensen PB. The Danish Psychiatric Central Register. Dan Med Bull 1997; 44:82-4.

34. Tebruegge M, Nandini V, Ritchie J. Does routine child health surveillance contribute to the early detection of children with pervasive developmental disorders? An epidemiological study in Kent, U.K. BMC Pediatr 2004;4:4

35. Chakrabarti S, Fombonne E. Pervasive developmental disorders in preschool children: confirmation of high prevalence. Am J Psychiatry 2005;162:1133-41.

36. Centers of Disease Control and Prevention. MMWR. Prevalence of autism spectrum disorders. Surveillance summaries. MMWR 2007:56:1-11.

37. Schendel D, Bhasin TK. Birth weight and gestational age characteristics of children with autism, including a comparison with other developmental disabilities. Pediatrics 2008;121:1155-64.

38. Gardener H, Spiegelman D, Buka SL. Prenatal risk factors for autism: comprehensive meta-analysis. Br J Psychiatry 2009:195:7-14.

39. Hvidtjorn D, Grove J, Schendel DE, et al. Cerebral palsy among children born after in vitro fertilization: the role of preterm delivery-a population-based, cohort study. Pediatrics 2006:118:475-82.

40. Lauritsen MB, Jørgensen M, Madsen KM, et al. Quality of childhood autism in the Danish psychiatric central register findings from a cohort sample born 1990-1999. J Autism Dev Disord. 2010:40:139-48. 